\title{
Existence and Stability of Periodic Solutions for Reaction-Diffusion Equations in the Two-Dimensional Case
}

\author{
Nefedov N. N., Nikulin E. I. \\ Received May 20, 2016
}

\begin{abstract}
Parabolic singularly perturbed problems have been actively studied in recent years in connection with a large number of practical applications: chemical kinetics, synergetics, astrophysics, biology, and so on. In this work a singularly perturbed periodic problem for a parabolic reaction-diffusion equation is studied in the two-dimensional case. The case when there is an internal transition layer under unbalanced nonlinearity is considered. The internal layer is localised near the so called transitional curve. An asymptotic expansion of the solution is constructed and an asymptotics for the transitional curve is determined. The asymptotical expansion consists of a regular part, an interior layer part and a boundary part. In this work we focus on the interior layer part. In order to describe it in the neighborhood of the transition curve the local coordinate system is introduced and the stretched variables are used. To substantiate the asymptotics thus constructed, the asymptotic method of differential inequalities is used. The upper and lower solutions are constructed by sufficiently complicated modification of the asymptotic expansion of the solution. The Lyapunov asymptotical stability of the solution was proved by using the method of contracting barriers. This method is based on the asymptotic comparison principle and uses the upper and lower solutions which are exponentially tending to the solution to the problem. As a result, the solution is locally unique.
\end{abstract}

The article is published in the authors' wording.

Keywords: reaction-diffusion, singular perturbations, small parameter, interior layers, unbalanced reaction, boundary layers, differential inequalities, upper and lower solutions

For citation: Nefedov N. N., Nikulin E. I., "Existence and Stability of Periodic Solutions for Reaction-Diffusion Equations in the Two-Dimensional Case", Modeling and Analysis of Information Systems, 23:3 (2016), 342-348.

On the authors:

Nefedov Nikolay Nikolaevich, Professor

Lomonosov Moscow State University,

GSP-1, 1-2 Leninskiye Gory, Moscow, 119991, Russia, e-mail: nefedov@phys.msu.ru

Nikulin Egor Igorevich, orcid.org/0000-0003-3850-4960, graduate student,

Lomonosov Moscow State University,

GSP-1, 1-2 Leninskiye Gory, Moscow, 119991, Russia, e-mail: nikulin@physics.msu.ru

Acknowledgments:

This work was supported by RFBR and RFBR-DFG projects (pr. 15-01-04619, 14-01-91333 ). 


\section{Introduction}

The main objective of the paper is the development and application of the asymptotic comparison principle in a new class of problems for nonlinear parabolic singularly perturbed equations in the two-dimensional case. These equations can have solutions with boundary and internal layers. These problems have been actively studied in recent years in connection with a large number of practical applications: chemical kinetics, synergetics, astrophysics, biology, and so on. Solutions of these problems, under natural assumptions, have narrow domains of rapid change, namely, boundary and internal layers, and they are difficult both for numerical solution and for asymptotic search.

\section{Problem statement}

In this work we consider a singularly perturbed problem. It is a parabolic nonlinear differential reaction-diffusion equation:

$$
\begin{aligned}
& N_{\varepsilon}(u):=\varepsilon^{2}\left(\triangle u-\frac{\partial u}{\partial t}\right)-F(u, x, y, t, \varepsilon)=0, \\
& (x, y, t) \in D_{t}:=\left\{(x, y, t) \in R^{3}:(x, y) \in D, t \in R\right\}, \\
& \frac{\partial u}{\partial n_{\Gamma}}(x, y, t, \varepsilon)=0, \quad(x, y) \in \Gamma, t \in R, \\
& u(x, y, t, \varepsilon)=u(x, y, t+T, \varepsilon), \quad(x, y) \in \bar{D}, t \in R,
\end{aligned}
$$

where $\triangle=\frac{\partial^{2}}{\partial x^{2}}+\frac{\partial^{2}}{\partial y^{2}}$, and the derivative $\frac{\partial}{\partial n_{\Gamma}}$ is taken along the inner normal to the smooth boundary $\Gamma$ of the given two-dimensional domain $D$, and $\varepsilon>0$ is a small parameter.

Assume that the following conditions hold:

(A1) Let $F(u, x, y, t, \varepsilon)$ be a function sufficiently smooth with respect to its arguments and T-periodic with respect to $t$.

(A2) The degenerate equation $F(u, x, y, t, 0)=0$ has precisely three isolated solutions T-periodic with respect to $t, \varphi^{(-)}(x, y, t)<\varphi^{(0)}(x, y, t)<\varphi^{(+)}(x, y, t), \quad(x, y, t) \in \bar{D}_{t}$.

(A3) $F_{u}\left(\varphi^{( \pm)}, x, y, t, 0\right)>0, F_{u}\left(\varphi^{(0)}, x, y, t, 0\right)<0, \quad(x, y, t) \in \bar{D}_{t}$. Using in the standard way the local system of coordinate $(r, \theta)$ near the curve $C_{0}$ (how to find this curve is explained below), let us introduce a new function

$$
I(r, \theta, t):=\int_{\varphi^{(-)}(r, \theta, t)}^{\varphi^{(+)}(r, \theta, t)} F(u, r, \theta, t, 0) d u .
$$

and impose the following conditions:

(A4) Suppose there exists a sufficiently smooth simple closed curve $C_{0}(t)$ lying in $D$, such that in the considered domain $\left.I\right|_{r=0}=0, \theta \in\left[0 ; \Theta_{0}\right]$, where $\left[0 ; \Theta_{0}\right]$ is the domain of variation of the coordinate $\theta$ on the curve $C_{0}$.

(A5) $\left.\frac{\partial I}{\partial r}\right|_{r=0}<0$, which means that the nonlinearity is unbalanced.

We search for the interface curve $C(t, \varepsilon)$ in the following form: $r=\lambda^{*}(\theta, t, \varepsilon)$.

As is known (see [15], [11], [10], [1]), problems similar to (1) can have solutions both with boundary layers near $\Gamma$ and the internal transition layers. In the present paper, 
we consider a solution such that, for every time moment $t$, as $\varepsilon$ tends to 0 , it tends to the root $\varphi^{(+)}(x, y, t)$ in the domain bounded by some smooth closed curve $C(t, \varepsilon) \subset D$ and tends to another root $\varphi^{(-)}(x, y, t)$ in the other part of the domain $D$. A domain of rapid change of the solution occurs in a neighborhood of the curve $C(t, \varepsilon)$, and the solution in this domain is referred to as an internal transition layer. Solutions of this kind are called contrast structures and the curve $C(t, \varepsilon)$ is the curve of the transition layer, or the interface curve; its position is not known in advance. Let us define the position of the curve of the transition layer by the condition of intersection of the solution and the root of the degenerate equation $\varphi^{(0)}(x, y, t), C(t, \varepsilon)=\left\{(x, y) \in D: \varphi^{(0)}(x, y, t)=u(x, y, t, \varepsilon)\right\}$. The curve $C(t, \varepsilon)$ partitions the domain $D$ into subdomains $D^{(-)}$and $D^{(+)}$which are correspondingly the outer and the inner domains for the curve.

\section{Construction of the asymptotic}

\subsection{The asymptotics for the solution}

A formal asymptotical expansion was constructed in the following form: $U^{( \pm)}\left(x, y, t, \varepsilon ; \lambda^{*}\right)=\bar{u}^{( \pm)}(x, y, t, \varepsilon)+Q^{( \pm)}\left(\tau, \theta, t, \varepsilon ; \lambda^{*}\right)+\Pi\left(\xi, \theta_{\Gamma}, t, \varepsilon\right)$, where the regular part is $\bar{u}^{( \pm)}(x, y, t, \varepsilon)=\bar{u}_{0}^{( \pm)}(x, y, t)+\varepsilon \bar{u}_{1}^{( \pm)}(x, y, t)+\ldots+\varepsilon^{n} \bar{u}_{n}^{( \pm)}(x, y, t)+\ldots$, the boundary layer part is $\Pi\left(\xi, \theta_{\Gamma}, t, \varepsilon\right)$ where $\xi=\frac{r_{\Gamma}}{\varepsilon}$ (here $\left(r_{\Gamma}, \theta_{\Gamma}\right)$ - a local system of coordinates for the curve $\Gamma$ ) and the interior layer part is

$$
Q^{( \pm)}\left(\tau, \theta, t, \varepsilon ; \lambda^{*}\right)=Q_{0}^{( \pm)}\left(\tau, \theta, t ; \lambda^{*}\right)+\varepsilon Q_{1}^{( \pm)}\left(\tau, \theta, t ; \lambda^{*}\right)+\ldots+\varepsilon^{n} Q_{n}^{( \pm)}\left(\tau, \theta, t ; \lambda^{*}\right)+\ldots
$$

where $\tau=\left(r-\lambda^{*}(\theta, t, \varepsilon)\right) / \varepsilon$. The part $Q^{( \pm)}\left(\tau, \theta, t, \varepsilon ; \lambda^{*}\right)$ depends on $\lambda^{*}$, but in general case is not a function depending on $\lambda^{*}$, thats why the notation "; "is used.

The method of boundary layer functions (see [6]), taking into account the specific features of a parabolic operator (see [1] and [15]), leads to a sequence of problems for determination of the coefficients of the asymptotic series (3), from which, in particular, we see that $\bar{u}_{0}^{(-)}(x, y, t)=\varphi^{(-)}(x, y, t), \bar{u}_{0}^{(+)}(x, y, t)=\varphi^{(+)}(x, y, t)$. The functions $\bar{u}_{i}^{( \pm)}(x, y, t)$, $i=1,2,3, \ldots$, and also the boundary layer functions $\Pi_{i}\left(\xi, \theta_{\Gamma}, t\right)$, are constructed in the standard way, and we do not consider this construction in the present paper.

For the part of the internal layer in zero approximation we have problems

$$
\begin{aligned}
& \frac{\partial^{2} Q_{0}^{( \pm)}\left(\tau, \theta, t ; \lambda^{*}\right)}{\partial \tau^{2}}=F\left(\varphi^{( \pm)}\left(\lambda^{*}, \theta, t\right)+Q_{0}^{( \pm)}\left(\tau, \theta, t ; \lambda^{*}\right), \lambda^{*}, \theta, t, 0\right) \\
& Q_{0}^{( \pm)}\left(0, \theta, t ; \lambda^{*}\right)+\bar{u}_{0}^{( \pm)}\left(\lambda^{*}, \theta, t\right)=\varphi^{(0)}\left(\lambda^{*}, \theta, t\right) \\
& Q_{0}^{( \pm)}\left( \pm \infty, \theta, t ; \lambda^{*}\right)=0 .
\end{aligned}
$$

wich have unique solutions monotone with respect to $\tau$. 
In the next approximation, we obtain the linear problems

$$
\begin{aligned}
& \frac{\partial^{2} Q_{1}^{( \pm)}}{\partial \tau^{2}}-\frac{\partial \tilde{F}^{*}}{\partial u} Q_{1}^{( \pm)}=r_{1}^{( \pm)}, \\
& Q_{1}^{( \pm)}\left(0, \theta, t ; \lambda^{*}\right)+\bar{u}_{1}^{( \pm)}\left(\lambda^{*}, \theta, t\right)=0, \\
& Q_{1}^{( \pm)}\left( \pm \infty, \theta, t ; \lambda^{*}\right)=0, \\
& r_{1}^{( \pm)}\left(\tau, \theta, t ; \lambda^{*}\right):=-\frac{\partial Q_{0}^{( \pm)}}{\partial \tau}\left(\tau, \theta, t ; \lambda^{*}\right) s\left(\lambda^{*}, \theta, t\right)+\tau\left(\frac{\partial \tilde{F}^{*}}{\partial u} \frac{\partial \bar{u}_{0}^{( \pm)}}{\partial r}\left(\lambda^{*}, \theta, t\right)+\frac{\partial \tilde{F}^{*}}{\partial r}\right)+ \\
& +\bar{u}_{1}\left(\lambda^{*}, \theta, t\right) \frac{\partial \tilde{F}^{*}}{\partial u}+\frac{\partial \tilde{F}^{*}}{\partial \varepsilon},
\end{aligned}
$$

where $s(r, \theta, t)=\triangle r(r, \theta, t)-\left.\frac{\partial r}{\partial t}(r, \theta, t)\right|_{x, y=\text { const }}$, the symbols $" \sim ", " * *$ above and to the right of a function mean that the value of the function is taken at the argument $\left(\varphi^{( \pm)}\left(\lambda^{*}, \theta, t\right)+Q_{0}^{( \pm)}\left(\tau, \theta, t ; \lambda^{*}\right), \lambda^{*}, \theta, t, 0\right)$. The solution of problems (3) is represented in an explicit form (see [15]).

Q-functions of subsequent orders are defined in a similar way.

\subsection{The asymptotics for the interface curve}

The asymptotics for the interface curve we will search in the following form:

$$
r=\lambda^{*}(\theta, t, \varepsilon)=\varepsilon \lambda_{1}^{*}(\theta, t, \varepsilon)=\varepsilon\left(\lambda_{1}(\theta, t)+\varepsilon \lambda_{2}(\theta, t)+\varepsilon^{2} \lambda_{3}(\theta, t)+\ldots\right) .
$$

The coefficients of the series are found from the condition of $C^{1}$-matching of the asymptotics on the curve $C$,

$$
\left.\varepsilon \frac{\partial U^{(+)}}{\partial r}\right|_{r=\lambda^{*}(\theta, t, \varepsilon)}=\left.\varepsilon \frac{\partial U^{(-)}}{\partial r}\right|_{r=\lambda^{*}(\theta, t, \varepsilon)}
$$

By substituting the asymptotics into condition and equating to zero the terms before every power of $\varepsilon$, we obtain the tasks for determining $\lambda_{i}$. Namely, for $\lambda_{1}(\theta, t)$ we have the following problem:

$$
\frac{\partial \hat{I}}{\partial r} \lambda_{1}+\int_{-\infty}^{+\infty}\left(-\Phi(\tau, \theta, t ; 0) \hat{s}+\frac{\partial \tilde{F}}{\partial r} \tau+\frac{\partial \tilde{F}}{\partial \varepsilon}\right) \Phi(\tau, \theta, t ; 0) d \tau=0
$$

Here the symbol " $\wedge$ " above a function mean that the value of the function is taken at the argument $(0, \theta, t)$. The coefficients $\lambda_{i}(\theta, t), i=2,3, \ldots$ are determined from the analogous tasks

$$
\frac{\partial \hat{I}}{\partial r} \lambda_{i}+f_{i}=0, \quad i \geq 1
$$

By virtue of assumption (A5) problems (6), (7) have the unique solutions. 


\section{Substantiation of the asymptotics thus constructed}

We denote by $U_{n}^{( \pm)}\left(x, y, t, \varepsilon ; \lambda^{*}\right)$ the partial sums of order $n$ of the asymptotic series constructed above in which the argument $\tau$ of the Q-functions is replaced by $\tau_{n}=$ $\left(r-\sum_{i=1}^{n+1} \varepsilon^{i} \lambda_{i}(\theta, t)\right) / \varepsilon$ and $\lambda^{*}$ by $\lambda_{n}^{*}=\sum_{i=1}^{n+1} \varepsilon^{i} \lambda_{i}(\theta, t)$ In the $\bar{D}_{n}^{(-)}$and $\bar{D}_{n}^{(+)}$into which the domain $\bar{D}$ is partitioned by the curve $r=\lambda_{n}^{*}$ when constructing $U_{n}^{( \pm)}\left(x, y, t, \varepsilon ; \lambda^{*}\right)$, we use the functions $Q^{(-)}$and $Q^{(+)}$, respectively. The following theorem holds.

Theorem 1. If conditions (A1)-(A5) hold, then, for sufficiently small $\varepsilon$, there is a solution $u(x, y, t, \varepsilon)$ of problem (1) which is a contrast structure of step type, and the following bound holds:

$$
\left|U_{n}\left(x, y, t, \varepsilon ; \lambda^{*}\right)-u(x, y, t, \varepsilon)\right|<C \varepsilon^{n+1}, \quad(x, y) \in \bar{D}, t \in R .
$$

The proof of this assertion is carried out using the asymptotic method of differential inequalities. We shall construct the upper and lower solutions of problem (1) by modifying the terms of the asymptotic series, similarly to the way in which it was done in [4]. For example, for the upper solution, one can take the function

$$
\begin{aligned}
& \beta_{n}(x, y, t, \varepsilon)=\bar{u}_{0}^{( \pm)}(x, y, t)+\varepsilon \bar{u}_{1}^{( \pm)}(x, y, t)+\ldots+\varepsilon^{n+2} \bar{u}_{n+2}^{( \pm)}(x, y, t) \\
& +Q_{0}^{( \pm)}\left(\tau_{\beta}, \theta, t ; \lambda_{n \beta}\right)+\varepsilon Q_{1}^{( \pm)}\left(\tau_{\beta}, \theta, t ; \lambda_{n \beta}\right)+\ldots+\varepsilon^{n+1} Q_{n+2}^{( \pm)}\left(\tau_{\beta}, \theta, t ; \lambda_{n \beta}\right) \\
& +\Pi_{\beta}\left(\xi, \theta_{\Gamma}, t, \varepsilon\right)+\varepsilon^{n+2}\left(\gamma+Q_{n+2, \beta}^{( \pm)}\left(\tau_{\beta}, \theta, t ; \lambda_{n \beta}\right)\right),
\end{aligned}
$$

where $\lambda_{n \beta}(\theta, t, \varepsilon)=\lambda_{n}^{*}(\theta, t, \varepsilon)+\varepsilon^{n+2}\left(\lambda_{n+2}(\theta, t, \varepsilon)-\nu(\theta, t)\right), \gamma>0$ is a constant ensuring the validity of the necessary differential inequality, and $\nu>0$ is a function ensuring the validity of the inequality for the jump of derivatives on the curve of transition layer. The functions $Q_{n+2, \beta}^{( \pm)}$are obtained by the scheme of [4] by modifying the equations for $Q_{n+2}^{( \pm)}$in which $\tau$ is replaced by $\tau_{\beta}=\left(r-\lambda_{n \beta}(t, \theta, \varepsilon)\right) / \varepsilon$, the functions $\Pi_{\beta}$ ensure the validity of the differential inequalities near $\Gamma$ and are not considered in this work. A lower solution $\alpha_{n}(x, y, t, \varepsilon)$ has a similar structure. All necessary inequalities are verified by the immediate calculations.

\section{Stability of periodic contrast structures}

The periodic solutions to the problem (1) can be considered as the solutions to corresponding boundary value problem with an initial condition:

$$
\begin{aligned}
& N_{\varepsilon}(v):=\varepsilon^{2}\left(\triangle v-\frac{\partial v}{\partial t}\right)-F(v, x, y, t, \varepsilon)=0, \\
& (x, y, t) \in D_{t+}:=\left\{(x, y, t) \in R^{3}:(x, y) \in D, 0<t<\infty\right\}, \\
& \frac{\partial u}{\partial n_{\Gamma}}(x, y, t, \varepsilon)=0, \quad(x, y) \in \Gamma, 0<t<\infty, \\
& v(x, y, 0, \varepsilon)=v^{0}(x, y), \quad(x, y) \in \bar{D} .
\end{aligned}
$$

Obviosly, if $v^{0}(x, y, \varepsilon)=u(x, y, 0, \varepsilon)$, where $u(x, y, t, \varepsilon)$ - the solution to the periodic problem (1), then the problem (8) also has the solution $v(x, y, t, \varepsilon)=u(x, y, t, \varepsilon)$. 
The problem of the Lyapunov stability of this solution is based on the asymptotical method of differential inequalities. We will seek upper and lower solutions to problem () in the form $\alpha(x, y, t, \varepsilon)=u(x, y, t, \varepsilon)+e^{-\lambda(\varepsilon) t}\left(\alpha_{n}(x, y, t, \varepsilon)-u(x, y, t, \varepsilon)\right), \beta(x, y, t, \varepsilon)=$ $u(x, y, t, \varepsilon)+e^{-\lambda(\varepsilon) t}\left(\beta_{n}(x, y, t, \varepsilon)-u(x, y, t, \varepsilon)\right)$, where $\lambda(\varepsilon)>0$ is sufficiently small. It can be shown that $N_{\varepsilon} \beta<0$ for $n \geq 0$. The inequality $N_{\varepsilon} \alpha>0$ can be checked similarly. Thus, the above constructed periodic solution is stable with the influence domain being at least $\left[\alpha_{0}, \beta_{0}\right]$.

Theorem 2. Let conditions (AO)-(A5) hold. Then the solution $u(x, y, t, \varepsilon)$ is Lyapunov asymptotically stable with the domain of stability containing at least $\left[\alpha_{0}, \beta_{0}\right]$, and hence, $u(x, y, t, \varepsilon)$ is a unique solution of problem (1) in this domain.

\section{References}

[1] N. N. Nefedov, L. Recke, K. R. Schnieder, "Existence and asymptotic stability of periodic solutions with an interior layer of reaction-advection-diffusion equations.", Journal of Mathematical Analysis and Applications, 405 (2013), 90-103.

[2] N. N. Nefedov, M. A. Davydova, "Contrast structures in singularly perturbed quasilinear reaction-diffusion-advection equations.", Differentsial'nye Uravneniya, 49 (2013), 715733.

[3] N.N. Nefedov, L. Recke, K.R. Schneider, "Asymptotic stability via the Krein-Rutman theorem for singularly perturbed parabolic periodic-Dirichlet problems.", Regular and Chaotic Dynamics, 15 (2010), 382-389.

[4] N.N. Nefedov, "The method of differential inequalities for some classes of nonlinear singularly perturbed problems with internal layers.", Differ. Uravn., 31 (1995), 11421149.

[5] V.T. Volkov and N.N. Nefedov, "Development of the asymptotic method of differential inequalities for investigation of periodic contrast structures in reacton-diffusion-advection equations.", Differ. Uravn., 46 (2006), 585-593.

[6] A.B. Vasil'eva and V. F. Butuzov, Asymptotic Expansions of the Solutions of Singularly Perturbed Equations (in Russian), Nauka, Moscow, 1973.

[7] P. Hess, Periodic-Parabolic Boundary Value Problems and Positivity, Pitman Research Notes in Math. Series 247, Longman Scientific\&Technical, 1991.

[8] D.H. Sattinger, Monotone methods in nonlinear elliptic and parabolic boundary value problems, Indiana Math. J. V. 21, N11, (1972), 979-1001.

[9] P. Fife, M. Tang, "Comparison principles for reaction-diffusion systems: Irregular comparison functions and applications to question of stability and speed propagation of front.", J. Diff. Equations., 40 (1981), 168-175.

[10] V.T. Volkov and N.N. Nefedov, "O periodicheskikh resheniyakh s pogranichnymi sloyami odnoy singulyarno vozmushchennoy modeli reaktsiya-diffuziya.", Computational Mathematics and Mathematical Physics, 34 (1994), 1307-1315.

[11] N. N. Nefedov, "Development of the Asymptotic Method of Differential Inequalities for Investigation of Periodic Contrast Structures in Reaction-Diffusion Equations.", Computational Mathematics and Mathematical Physics, 46 (2006), 614-622.

[12] N. N. Nefedov, "Comparison Principle for Reaction-Diffusion-Advection Problems with Boundary and Internal Layers.", Lecture Notes in Computer Science, 8236 (2013), 62-72.

[13] N. N. Nefedov, O. E. Omel'chenko, "Periodic Step-Like Contrast Structures for a Singularly Perturbed Parabolic Equation.", Differ. Uravn., 36 (2000), 209-218.

[14] N. N. Nefedov, "An Asymptotic Method of Differential Inequalities for the Investigation of Periodic Contrast Structures: Existence, Asymptotics, and Stability.", Differ. Uravn., 36 (2000), 262-269. 
[15] A. B. Vasil'eva, V.F. Butuzov, N. N. Nefedov, "Kontrastnye struktury v singulyarno vozmushchennykh zadachakh.", Fundamental'naya i prikladnaya matematika, 4 (1998), 799-851.

[16] C.V. Pao, Nonlinear parabolic and elliptic equations, Plenum Press, New York and London, 1992.

[17] Amman H. Periodic solutions of semilinear parabolic equations in nonlinear analysis New York: Acad. Press, 1978.

Нефедов Н. Н., Никулин Е. И., "Существование и устойчивость периодических решений уравнения реакция-диффузия в двумерном случае", Моделирование и анализ информационых систем, 23:3 (2016), 342-348.

DOI: $10.18255 / 1818-1015-2016-3-342-348$

Аннотация. Параболические сингулярно возмущенные задачи активно исследуются в последние годы в связи с большим количеством практических применений: химическая кинетика, синергетика, астрофизика, биология и т.д. В этой работе исследуется сингулярно возмущенная периодическая задача для параболического уравнения реакция-диффузия в двумерном случае. Рассматривается случай существования внутреннего переходного слоя при несбалансированной нелинейности. Внутренний слой локализован вблизи так называемой кривой переходного слоя. Строится асимптотическое разложение решения и определяется асимптотика для кривой переходного слоя. Асимптотическое разложение состоит из регулярной части, внутреннего слоя и части пограничного слоя. В этой работе мы сфокусируем внимание на части внутреннего переходного слоя. С целью его описания вводится локальная система координат в окрестности кривой перехода и используются растянутые переменные. Чтобы обосновать таким образом построенную асимптотику, используется асимптотический метод дифференциальных неравенств. Верхнее и нижнее решения строятся путем достаточно сложной модификации асимптотического разложения решения. Асимптотическая устойчивость решения по Ляпунову доказывается с помощью метода сужающихся барьеров. Этот метод базируется на принципе дифференциальных неравенств, и в нем используются верхнее и нижнее решения, которые экспоненциально стремятся к решению задачи. Как результат, решение является локально единственным.

Статья публикуется в авторской редакции.

Ключевые слова: реакция-диффузия, сингулярные возмущения, малый параметр, внутренние слои, несбалансированная реакция, пограничные слои, дифференциальные неравенства, верхние и нижние решения

\section{Об авторах:}

Нефедов Николай Николаевич, д-р. физ.-мат. наук, профессор,

Московский государственный университет им. М.В. Ломоносова

ул. Ленинские горы, д. 1, стр. 2, г. Москва, 119991 Россия, e-mail: nefedov@phys.msu.ru

Никулин Егор Игоревич, orcid.org/0000-0003-3850-4960, аспирант,

Московский государственный университет им. М.В. Ломоносова,

ул. Ленинские горы, д. 1, стр. 2, г. Москва, 119991 Россия, e-mail: nikulin@physics.msu.ru

Благодарности:

Работа выполнена при финансовой поддержке РФФИ и РФФИ - ННИО (проекты 15-01-04619, 14-01-91333 ). 